\title{
Acute portal vein thrombosis secondary to COVID- 19: a case report
}

Roham Borazjani

Student Research Committee, Shiraz University of Medical Sciences, Shiraz, Iran

\section{Seyed Reza Seraj}

Department of Internal Medicine, Shiraz University of Medical Sciences, Shiraz, Iran

\section{Mohammad javad Fallahi ( $D$ fallahimj@sums.ac.ir)}

Thoracic and Vascular surgery research Center, Shiraz University of Medical Sciences, Shiraz, Iran https://orcid.org/0000-0002-8562-5637

\section{Zhila Rahmanian}

Department of Internal Medicine, Jahrom University of Medical Sciences, Jahrom, Iran

\section{Case Report}

Keywords: COVID-19, Pneumonia, Portal vein, Thrombosis

Posted Date: July 2nd, 2020

DOI: https://doi.org/10.21203/rs.3.rs-39171/v1

License: (c) (1) This work is licensed under a Creative Commons Attribution 4.0 International License. Read Full License 


\section{Abstract}

Introduction: COVID-19 pneumonia exhibits several extra-pulmonary complications.

Case presentation: We described a 23-year old male with coronavirus pneumonia and portal vein thrombosis.

Conclusion: Clinicians should always consider thrombosis and other hypercoagulable diseases in patients with COVID-19.

\section{Introduction}

Novel Coronavirus pneumonia was first described as pneumonia of unknown cause in Wuhan, China, at the end of 2019(1) and rapidly became pandemic. With time, some new extra-pulmonary manifestations of this viral pneumonia were described. Increased incidence of thromboembolic events was frequently reported (2).

Herein, we aim to describe a 26-year-old male with COVID-19 pneumonia and acute Portal Vein Thrombosis (PVT).

\section{Case Presentation}

A 26-year-old male known case of asthma was brought to the Emergency Department (ED) in Faghihi hospital, Shiraz, Iran, on 11 April 2020 due to acute-onset dyspnea and a decrease in Level of Consciousness since the day of admission. He was admitted with the impression of an acute asthma attack. There was no history of fever, hemoptysis, diarrhea, nausea, and vomiting (N/V), lower gastrointestinal tract bleeding, incontinency, stroke signs, and symptoms. He had asthma for several years and only used the salbutamol inhaler as needed. His social history was positive for alcohol, cigarette smoking, and occasionally marijuana.

We intubated the patient due to severe hypoxia and respiratory distress; blood Oxygen saturation $\left(\mathrm{O}_{2}\right.$ Sat.) was $60 \%$ and $97 \%$ before and after intubation, respectively. The initial vital sign was; temperature: 36.9 C, pulse rate: 110 / minute, respiratory rate: $36 /$ minute, and blood pressure: $120 / 90 \mathrm{mmHg}$. Generalized wheeze was heard through auscultation. Other physical findings were normal. Brain computed tomography (CT) was normal. Chest CT showed bilateral peripheral and peribronchovascular patchy ground-glass opacities in both lung fields with tree-in-bud appearance. (Figure1).The tracheal aspirate was positive for real-time polymerase chain reaction (RT-PCR) for SARS-COV2. The blood routine tests are summarized in table 1. 
11 April 15 April 16 April 21 April 29 April

\begin{tabular}{|c|c|c|c|c|c|c|}
\hline Tests & & & Result & & & Reference \\
\hline $\mathrm{WBC}\left(* 10^{3} / \mu \mathrm{L}\right)$ & 18.1 & 14.1 & 8.6 & 9.2 & 5.4 & $4.8-10.8$ \\
\hline $\mathrm{NE}(\%)$ & 68 & 93.3 & 90.5 & 85.5 & 65.3 & $40-80$ \\
\hline LY (\%) & 12 & 4.9 & 7.1 & 12.1 & 22.2 & $20-40$ \\
\hline $\mathrm{Hb}(\mathrm{g} / \mathrm{dL})$ & 14.7 & 10.7 & 9.4 & 10.9 & 10 & $13.5-18$ \\
\hline $\operatorname{Plt}\left(* 10^{9} / \mathrm{L}\right)$ & 213 & 93 & 78 & 127 & 171 & $150-450$ \\
\hline PT (sec) & 19.2 & & 17.2 & 20.1 & & $11-14$ \\
\hline aPTT(sec) & 28 & & 29 & 29 & & $27-35$ \\
\hline $\mathrm{Cr}(\mathrm{mg} \%)$ & 1.3 & 1 & 0.8 & 0.7 & 17 & $07-1.4$ \\
\hline BUN (mg \%) & 21 & 21 & 21 & 18 & 1 & $6-23$ \\
\hline f.Alb (gr/dL) & & & & 1.6 & & \\
\hline f.pro(gr/dL) & & & & 2.7 & & \\
\hline $\operatorname{AST}(\mathrm{U} / \mathrm{L})$ & & & & 44 & 40 & $2-37$ \\
\hline $\mathrm{ALT}(\mathrm{U} / \mathrm{L})$ & & & & 67 & 45 & $2-41$ \\
\hline Alk.p(U/L) & & & & 138 & 138 & 64- 306 \\
\hline Amylase (U/L) & & & & 42 & 42 & $20-100$ \\
\hline Lipase (U/L) & & & & 15 & 17 & $5-60$ \\
\hline T.B(mg \%) & & & & 1.41 & 2.44 & $0.1-1.2$ \\
\hline D.B (mg \%) & & & & 0.27 & 0.98 & $0-0.3$ \\
\hline Serum Alb (gr/dL) & & & & & 2.8 & $3.5-5.3$ \\
\hline Serum protein $(\mathrm{gr} / \mathrm{dL})$ & & & & & 4.6 & $6.6-8.6$ \\
\hline $\operatorname{TIBC}(\mu \mathrm{g} / \mathrm{dL})$ & & & & 277 & & $230-440$ \\
\hline $\mathrm{sFe}(\mu \mathrm{g} / \mathrm{dL})$ & & & & 49 & & $40-168$ \\
\hline
\end{tabular}

Table 1. A breif summery of blood routine tests. WBC, White Blood Cell; NE, Neutrophil; LY, Lymphocye; Hb; Hemoglobulin; Plt, Platelet; PT, Prothrombin Time; APTT, Activated Partial Thromboplastin Time; Cr, creatinine; BUN, Blood Urea Nitrogen Level; f.Alb, Albumin of ascitic fluid; f.pro, protein of ascitic fluid; AST, Aspartate transaminase; ALT, Alanine Transaminase; Alk.p, Alkaline Phosphatase; T.B, Total Bilirubin; D.B, Direct Bilirubin; TIBC, Total iron-binding capacity; sFe, Serum Iron.

Figure 1. Chest CT. The CT showed bilateral peripheral and peribronchovascular patchy ground-glass opacities (arrows) in both lungs with tree-in-bud appearance Electrocardiography showed sinus tachycardia, and other findings were normal. Urine analysis was positive for marijuana, benzodiazepine, and morphine. Venous Blood gas analysis was as follows; PH was 6.97, partial pressure of carbon dioxide $\left(\mathrm{PCO}_{2}\right) 103 \mathrm{mmHg}$, and venous bicarbonate level $\left(\mathrm{HCO}_{3}\right) 23 \mathrm{mmol} / \mathrm{L}$. We started initial treatment immediately with dexamethasone (4mg, IV every 8 hours), Piperacillin/tazobactam ( $3.375 \mathrm{~g}$, IV every 6 hours), prophylactic heparin (5000 IU every 12 hours). Due to significant patient-ventilator asynchrony, the patient was paralyzed with cisatracurium in addition to midazolam and propofol. During the mechanical ventilation course, he developed progressive thrombocytopenia (table1), but no apparent cause was found. Therefore, intravenous immunoglobulin ( $25 \mathrm{gr}$ per day for three days) was prescribed for 
presumed SARS-COV2-induced thrombocytopenic purpura. His respiratory condition got better during the next five days when we started weaning the patient off the mechanical ventilation, and he was extubated on 16 April 2020, but thrombocytopenia persisted.

On 21 April 2020 (10 days after admission), the patient developed upper GI bleeding associated with generalized abdominal pain with distension. Upper GI endoscopy was normal except for the presence of minimal erythema and congestion in the cardia and fundus. No esophageal and fundal varices and duodenal ulcers were reported. Abdominopelvic Sonography showed mild free fluid in the abdominal cavity. The diagnostic abdominal tap was done, and the results are summarized in table2.

Abdominal CT showed hypoperfused areas in the posterior segment of the right (Rt.) hepatic lobe and the medial and lateral segments of left (Lt.) lobe along with evidence of filling defect in main Rt. Portal vein. Also, Lt. portal vein was not opacified(Figure2). Color Doppler Sonography (CDS) of the abdomen showed the absence of flow in the portal vein without collateral veins consistent with acute portal vein thrombosis. An extensive evaluation of the possible underlying hypercoagulable state was unremarkable (Table 1 and 2).

\begin{tabular}{lcc} 
Tests & Result & Reference \\
\hline $\mathrm{C}_{3}$ & $130 \mathrm{mg} / \mathrm{dL}$ & Adults: $90-180 \mathrm{mg} / \mathrm{dL}$ \\
\hline $\mathrm{C}_{4}$ & $29 \mathrm{mg} / \mathrm{dL}$ & $100-40 \mathrm{mg} / \mathrm{dL}$ \\
\hline $\mathrm{LAC}$ & $40.8 \mathrm{sec}$. & $28-42 \mathrm{sec}$ \\
\hline Protein C & $81 \%$ & $65-160 \%$ \\
\hline Protein S & $89.8 \%$ & $65-150 \%$ \\
\hline Factor V leiden & $91.6 \mathrm{sec}$ & $\geq 120 \mathrm{sec}$ \\
\hline Anti-thrombin & $123 \%$ & $70-140 \%$ \\
\hline ASMA & $\mathrm{N}$ & Up to $1: 10$ \\
\hline ANA & $7 \mathrm{U} / \mathrm{mL}$ & Negative: $<12$ \\
& & Borderline: $12-18$ \\
& & Positive: $>18$ \\
\hline Anti ds-DNA & $1.3 \mathrm{U} / \mathrm{mL}$ & Negative: $<12$ \\
& & Borderline: $12-18$ \\
& & Positive: $>18$ \\
\hline$\beta_{2}$ glyco-IgG & $52.8 \mathrm{U} / \mathrm{mL}$ & Negative: $<12$ \\
& & Equivocal: $12-18$ \\
\hline$\beta_{2}$ glyco-IgM & $2.4 \mathrm{U} / \mathrm{mL}$ & Positive: $>18$ \\
\hline & & Negative: $<12$ \\
& & Positive: $>18$
\end{tabular}

Table2. Laboratory findings in the patient. LAC, Lupus Anticoagulant; ASMA, Anti-smooth Muscle Antibody; ANA, Antinuclear Antibody; $\beta_{2}$ glyco-IgG, beta-2 glycoprotein immunoglobulin $G$ antibody; $\beta_{2}$ glyco-IgM, beta-2 glycoprotein immunoglobulin $M$ antibody. 
Figure 2. Abdominopelvic CT with IV contrast. Hypoperfusion is seen in the posterior segment of the right (Rt.) hepatic lobe and the medial and lateral segments of the left (Lt.) lobe. Evidence of filling defect in the main Rt. portal vein is also detected (arrows).

When gastrointestinal bleeding was stopped, Enoxaparin was started at a dose of $60 \mathrm{IU}$ every 12 hours. After about one week, his abdominal pain well improved, and platelet count increased, and finally, the patient was discharged home.

\section{Discussion}

Herein, we report a coincidence of the acute portal vein thrombosis and COVID-19 respiratory pneumonia in the background of an acute asthma attack. PVT is a rare venous thromboembolic disease typically occurred in patients with an underlying disease such as decompensated cirrhosis and/or malignancies, pancreatitis, Systemic lupus erythematosus, and other hypercoagulable states(2). Our patient did not have any of the mentioned risk factors. There is some not proven concern about the increased thrombotic event during or early after IV immunoglobulin exists. Still, no episode of portal vein thrombosis is linked to IVIG to date(3).

Several studies showed that thromboembolic events increases in patients with SARS-CoV-2 infection since the pandemic declared by the World Health Organization (WHO)(4-6). In the previous studies, thromboembolic events were pulmonary emboli, deep venous thrombosis (DVT), and cerebral infarction usually occurred in the elderly with severe COVID-19 and comorbid diseases such as hypertension and diabetes $(4,6,7)$. To our knowledge, our patient is the second case with PVT. The first was a 79-year old female without unknown medical comorbidities who developed with diarrhea and epigastric abdominal pain and acute dyspnea eight days after abdominal pain. However, in our study, the patient was a young man without any significant comorbidities except for a history of asthma and chronic alcohol consumption.

The exact pathophysiology of the increased prevalence of thromboembolism events in COVID-19 positive patients remains unclear(4). But, immobilization and hypoxemia, as seen in our patient at the time of admission along with the increase in inflammatory cytokines such as interleukin-6 (IL-6), IL-10, and Tumor Necrosis Factor Alfa (TNF-a) are the possible mechanisms (8-10). Unfortunately, we cannot assess the serum level of these cytokines. The attachment of the viruses to the endothelial surfaces via angiotensin-converting enzyme receptor (ACE-R), leads to the lymphocytic endothilitis, interferon-1 (INF1), and prothrombotic genes overexpression(11). Becker speculated that the attachment of COVID-19related extracellular RNA to the factor $\otimes$ and $\otimes$ which belongs to the contact pathway of the coagulation cascade leads to the interferon- $\gamma$ (INF- $\gamma$ ) suppression and a hyper-inflammatory state(12)

Our report of portal vein thrombosis in the setting of COVID-19 pneumonia add portal vein thrombosis to other COVID-19 associated thrombotic phenomenon and denote that clinician who is caring these patients should have a high index of suspicion to diagnose this unusual presentation in a proper clinical scenario. 


\section{List Of Abbreviations}

PVT: portal vein thrombosis

ED: emergency department

N/V: Nausea and vomiting

CT: Computed tomography

CXR: chest X-ray

RT-PCR: real-time polymerase chain reaction

Rt.: right

Lt.: left

CDS: color doppler sonography

WHO: world health organization

DVT: deep venous thrombosis

IL: interleukin

ACE-R: angiotensin-converting enzyme receptor

INF: interferon

\section{Declarations}

Ethics approval and consent to participate: This case report was conducted in accordance with the Declaration of Helsinki. Ethical approval for this study was obtained from the Research Ethics Committee of Shiraz University of Medical Sciences.

Consent for publication: Written informed consent was obtained from the patient for publication of this case report and any accompanying images. A copy of the written consent is available for review by the Editor of this journal.

Availability of data and materials: The datasets analyzed during the current study are available from the corresponding author on reasonable request.

Competing interests: The authors declare that they have no competing interests

Funding: Not applicable 
Authors' contributions: R.B., R.S, M.F., and Z.M. contributed to the design and implementation of the research, to the analysis of the results and the writing of the manuscript. All Authors read and approved the manuscript.

Acknowledgments: Not applicable

\section{References}

1. Jiang F, Deng L, Zhang L, Cai Y, Cheung CW, Xia Z. Review of the clinical characteristics of coronavirus disease 2019 (COVID-19). Journal of General Internal Medicine. 2020:1-5.

2. Klok FA, Kruip M, van der Meer NJM, Arbous MS, Gommers D, Kant KM, et al. Confirmation of the high cumulative incidence of thrombotic complications in critically ill ICU patients with COVID-19: An updated analysis. Thromb Res. 2020.

3. Basta M. Intravenous immunoglobulin-related thromboembolic events - an accusation that proves the opposite. Clinical \& Experimental Immunology. 2014;178:153-5.

4. Zhou B, She J, Wang Y, Ma X. A case of coronavirus disease 2019 with concomitant acute cerebral infarction and deep vein thrombosis. Frontiers in Neurology. 2020;11.

5. Zhou B, She J, Wang Y, Ma X. Venous thrombosis and arteriosclerosis obliterans of lower extremities in a very severe patient with 2019 novel coronavirus disease: a case report. Journal of Thrombosis and Thrombolysis.1.

6. Bozzani A, Arici V, Franciscone MM, Danesino V, Cascina A, Ticozzelli G, et al. Severe Acute Respiratory Syndrome Coronavirus 2 Infection and the Upper Limb Deep Vein Thrombosis Risk. Ann Vasc Surg. 2020.

7. Cui S, Chen S, Li X, Liu S, Wang F. Prevalence of venous thromboembolism in patients with severe novel coronavirus pneumonia. J Thromb Haemost. 2020.

8. Huang C, Wang Y, Li X, Ren L, Zhao J, Hu Y, et al. Clinical features of patients infected with 2019 novel coronavirus in Wuhan, China. The lancet. 2020;395(10223):497-506.

9. Connors JM, Levy JH. Thromboinflammation and the hypercoagulability of COVID-19. Journal of Thrombosis and Haemostasis. 2020.

10. Barco S, Konstantinides SV, Investigators CT. Thrombosis and Thromboembolism Related to COVID19 A clarion call for obtaining solid estimates from large-scale multicenter data. Research and Practice in Thrombosis and Haemostasis.

11. Sharma A, Garcia G, Arumugaswami V, Svendsen CN. Human iPSC-Derived Cardiomyocytes are Susceptible to SARS-CoV-2 Infection. bioRxiv.

12. Becker RC. COVID-19 update: Covid-19-associated coagulopathy. Journal of Thrombosis and Thrombolysis. 2020.

\section{Figures}




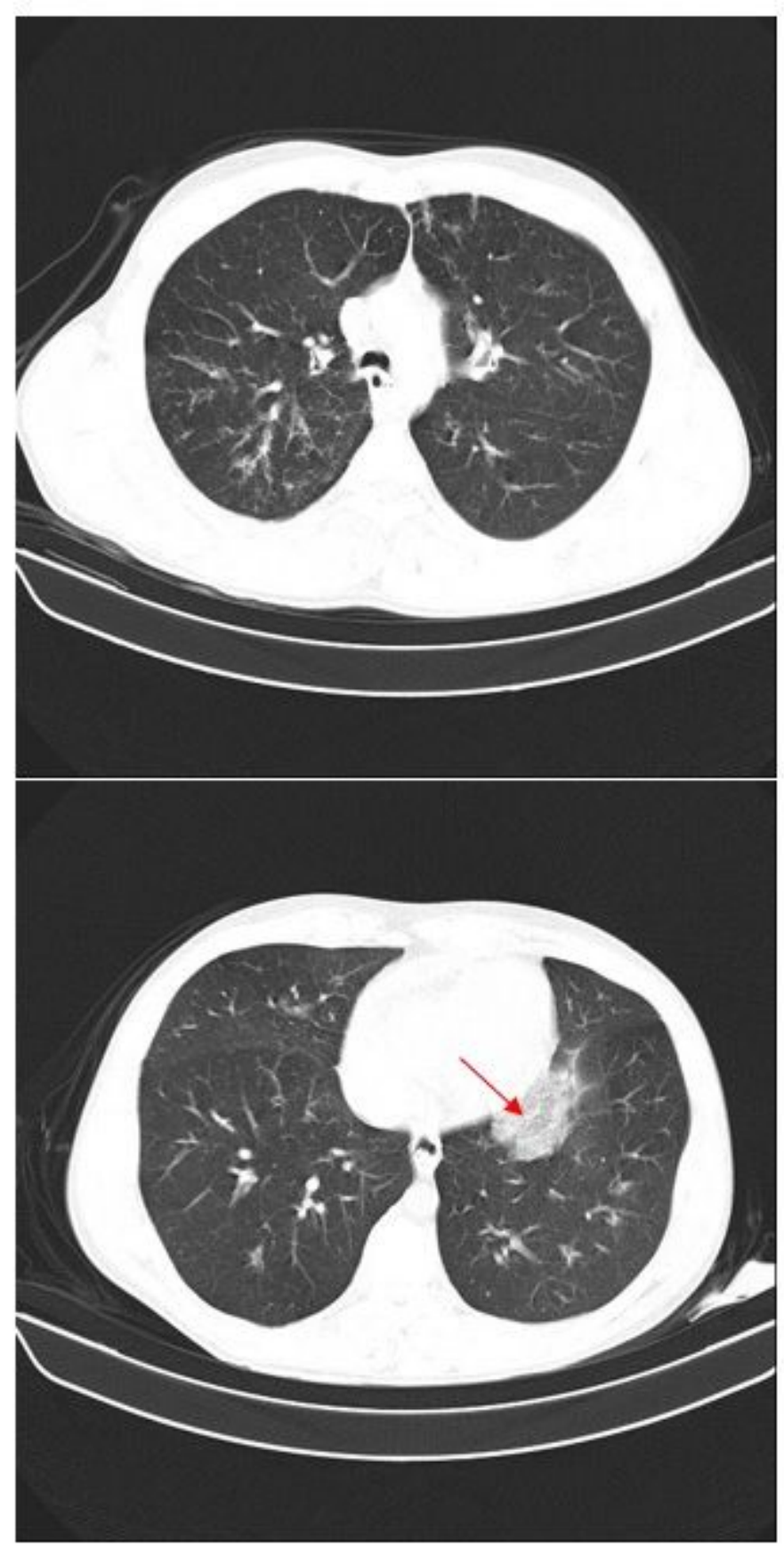

Figure 1

Chest CT. The CT showed bilateral peripheral and peribronchovascular patchy ground-glass opacities (arrows) in both lungs with tree-in-bud appearance 


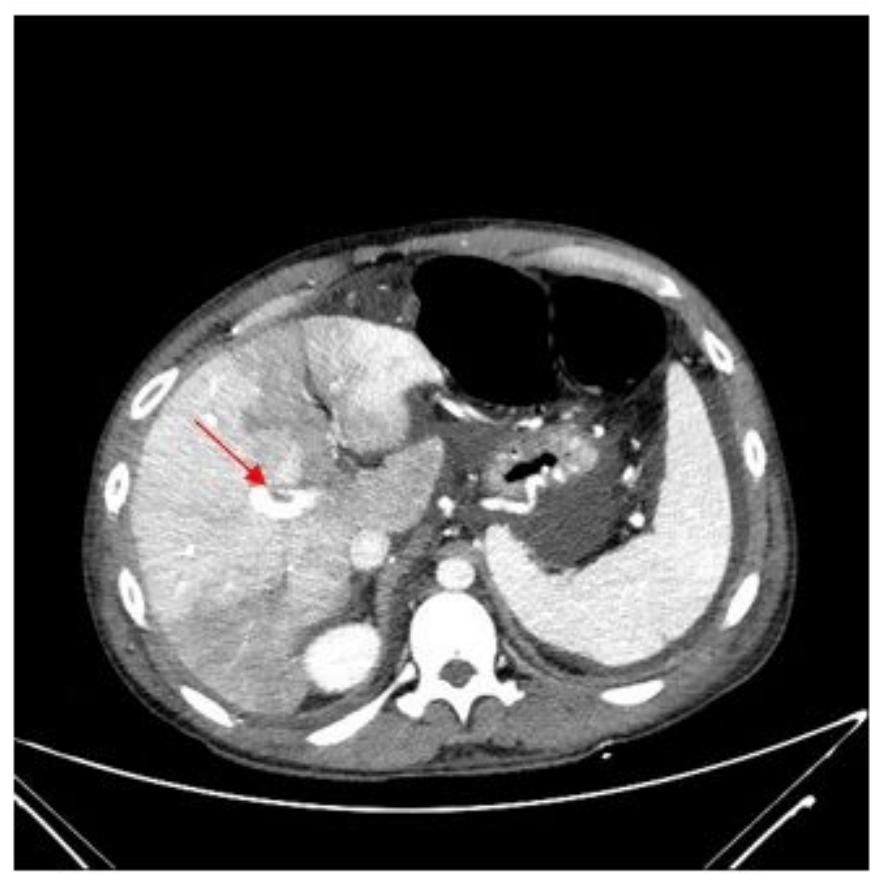

Figure 2

Abdominopelvic CT with IV contrast. Hypoperfusion is seen in the posterior segment of the right (Rt.) hepatic lobe and the medial and lateral segments of the left (Lt.) lobe. Evidence of filling defect in the main Rt. portal vein is also detected (arrows).

\section{Supplementary Files}

This is a list of supplementary files associated with this preprint. Click to download.

- CAREchecklistEnglish2013.pdf 